\title{
CARLESON MEASURES \\ IN HARDY AND WEIGHTED BERGMAN SPACES OF POLYDISCS
}

\author{
F. JAFARI
}

(Communicated by Paul S. Muhly)

\begin{abstract}
The importance of theorems on Carleson measures has been well recognized [3]. In [1] Chang has given a characterization of the bounded measures on $L^{p}\left(T^{n}\right)$ using what one may characterize as the bounded identity operators from Hardy spaces of polydiscs in $L^{p}$ spaces. In [4] Hastings gives a similar result for (unweighted) Bergman spaces of polydiscs. In this paper we characterize the bounded identity operators from weighted Bergman spaces of polydiscs into $L^{p}$ spaces, and classify those operators which are compact on the Hardy and weighted Bergman spaces in terms of Carleson-type conditions. We give two immediate applications of these results here, and a much broader class of applications elsewhere [5].
\end{abstract}

\section{DEFINITIONS AND PRELIMINARIES}

We denote by $U^{n}$ the unit polydisc in $C^{n}$, by $T^{n}$ the distinguished boundary of $U^{n}$, by $H^{p}$ the Hardy space of order $p$ in $U^{n}$, by $A_{\alpha}^{p}$ the weighted Bergman spaces of order $p$ with weights $\prod_{i=1}^{n}\left(1-\left|z_{i}\right|^{2}\right)^{\alpha}, \alpha>-1 . D_{\alpha}$ will be used to denote the weighted Dirichlet spaces with respect to these weights. We shall use $m_{n}$ to denote the $n$-dimensional Lebesgue area measure on $T^{n}$, normalized so that $m_{n}\left(T^{n}\right)=1$. By $\sigma_{n}$ we shall denote the volume measure on $\overline{U^{n}}$, defined so that $\sigma_{n}\left(\overline{U^{n}}\right)=1$, and by $\sigma_{n, \alpha}$ we shall denote the weighted measure on $\overline{U^{n}}$ given by $\prod_{i=1}^{n}\left(1-\left|z_{i}\right|^{2}\right)^{\alpha} \sigma_{n}$. We use $R$ to describe rectangles on $T^{n}$, and we use $S(R)$ to denote the corona associated to these sets. In particular, if $I$ is an interval on $T$ of length $\delta$ centered at $e^{i\left(\theta_{0}+\delta / 2\right)}$,

$$
S(I)=\left\{z \in U: 1-\delta<r<1, \theta_{0}<\theta<\theta_{0}+\delta\right\} .
$$

Then if $R=I_{1} \times I_{2} \times \cdots \times I_{n} \subset T^{n}$, with $I_{j}$ intervals having length $\delta_{j}$ and having centers $e^{i\left(\theta_{j}^{0}+\delta_{j} / 2\right)}, S(R)$ is given by $S(R)=S\left(I_{1}\right) \times S\left(I_{2}\right) \times \cdots \times S\left(I_{n}\right)$. We use $S$ to denote $S(R)$ whenever convenient. If $V$ is any open set in $T^{n}$ we define $S(V)=\bigcup_{\alpha} S\left(R_{\alpha}\right)$ where $\left\{R_{\alpha}\right\}$ runs through all rectangles in $V$. A

Received by the editors January 8, 1990.

1980 Mathematics Subject Classification (1985 Revision). Primary 47A30; Secondary 32A35.

Key words and phrases. Hardy and weighted Bergman spaces, Carleson measures, polydiscs. 
finite, nonnegative, Borel measure $\mu$ on $\overline{U^{n}}$ is said to be a (bounded) Carleson measure if $\mu(S(V)) \leq C m_{n}(V)$ for all connected open sets $V \subset T^{n} . \mu$ is said to be a compact-Carleson measure if

$$
\lim _{m_{n}(V) \rightarrow 0} \sup _{V \subset T^{n}} \frac{\mu(S(V))}{m_{n}(V)}=0 .
$$

$\mu$, or where appropriate $\mu_{\alpha}$, is said to be a (bounded) $\alpha$-Carleson measure if $\mu(S(R)) \leq C \prod_{i=1}^{n} \delta_{i}^{2+\alpha}$ for all $R \subset T^{n}$, and likewise $\mu$, or $\mu_{\alpha}$, is a compact $\alpha$-Carleson measure if

$$
\lim _{\delta_{i} \rightarrow 0} \sup _{\theta \in T^{n}} \frac{\mu(S(R))}{\prod_{i=1}^{n} \delta_{i}^{2+\alpha}}=0 .
$$

We point out that Carleson measures as they are used in the literature are always bounded. One should also note that the definitions of these measures have nothing to do with Hardy or weighted Bergman spaces. The definition of Carleson measures for polydiscs is due to Chang [1].

By considering the homogeneous expansion of $f$ it is possible to obtain the following characterizations of $A_{\alpha}^{2}$ and $D_{\alpha}$.

Proposition 1.1. For $\alpha>-1$

(i)

(ii)

$$
f \in A_{\alpha}^{2}\left(U^{n}\right) \Leftrightarrow \sum_{|s|=0}^{\infty}\left|a_{s}\right|^{2} \prod_{i=1}^{n}\left(s_{i}+1\right)^{-1-\alpha}<\infty
$$

$$
f \in D_{\alpha}\left(U^{n}\right) \Leftrightarrow \sum_{|s|=0}^{\infty}\left|a_{s}\right|^{2} \sum_{j=1}^{n} s_{j}^{2} \prod_{i=1}^{n}\left(s_{i}+1\right)^{-1-\alpha}<\infty,
$$

where $|s|=\sum_{i=1}^{n} s_{i}$ and multi-index notation is used.

An immediate consequence of this proposition and the definitions is

Corollary 1.2. For $0<p<\infty$ and $-1<\alpha<\beta$

(i) $D_{\alpha}\left(U^{n}\right) \subset A_{\alpha}^{2}\left(U^{n}\right)$,

(ii) $A_{\alpha}^{\alpha}\left(U^{n}\right) \subset A_{\beta}^{p}\left(U^{n}\right)$,

(iii) $D_{\alpha}\left(U^{n}\right) \subset D_{\beta}\left(U^{n}\right)$,

(iv) $A_{-1}^{2}\left(U^{n}\right)=H^{2}\left(U^{n}\right)$, and

(v) $A_{\alpha}^{2} \subset D_{\alpha+2}$.

The inclusions are strict.

We note that the equivalence that exists between weighted Bergman and other weighted Dirichlet spaces in the disc or the unit ball (see [8], for example) fails in polydiscs. In fact, to recover this equivalence we need to replace the weights by $\left(1-\|z\|^{2}\right)^{n \alpha}$ where $\|\cdot\|$ is the polydisc norm.

We shall show that whenever $\mu_{\alpha}$ is a finite, positive, Borel measure on $U^{n}$ of the type $\prod_{i=1}^{n}\left(1-\left|z_{i}\right|^{2}\right)^{\alpha} d \nu_{n}$, where $\nu_{n}$ is an arbitrary positive, Borel measure on $U^{n}$ (not depending on $\alpha$ ), then we have the following proposition. 
Proposition 1.3. Suppose $-1<\alpha<\beta$ and let $I_{\alpha}$ be the identity operator from $A_{\alpha}^{p}\left(U^{n}\right)$ into $L^{p}\left(\mu_{\alpha}\right)$. If $I_{\alpha}$ is a bounded (compact) operator, then so is $I_{\beta}$.

We shall prove this proposition at the end of the next section where we shall discuss the relationship between the operator $I_{\alpha}$ and a class of positive, finite, Borel measures on $\overline{U^{n}}$. In the proceeding, we shall frequently make use of the following two classical results [9, Example 18, p. 107]:

Lemma 1.4. (i) Let $X$ be a reflexive Banach space. Then an operator $T: X \rightarrow X$ is compact if and only if $T$ takes weakly convergent sequences to norm convergent sequences in $X$.

(ii) In $H^{p}$ and $A_{\alpha}^{p}, p>1$, weak convergence is equivalent to norm boundedness and pointwise convergence.

This lemma states that compactness of an operator $T$ on $H^{p}\left(U^{n}\right)$ or $A_{\alpha}^{p}\left(U^{n}\right)$ is equivalent to $T$ taking every norm bounded sequence $f_{n}$ converging to zero uniformly on compact subsets of $U^{n}$ to a sequence converging to zero in the appropriate norm.

This work forms part of the dissertation of the author written at the University of Wisconsin-Madison under the supervision of Professor Walter Rudin. The author wishes to thank Professor Walter Rudin for his careful and critical review of this work, and his assistance in writing these results.

\section{MAIN RESULtS}

Garnett [3] provides a long history of the development and application of Carleson measures. This rich area of research contains a large body of literature on characterizations of different classes of operators in different spaces and their applications. Chang [1] has characterized the bounded measures on $L^{p}\left(T^{2}\right)$ using a two-line proof referring to a result of Stein. Characterization of the bounded identity operators on Hardy spaces is an immediate consequence of Chang's proof using standard arguments. Hastings [4] has given a similar result for unweighted Bergman spaces. Recently MacCluer [7] has obtained a Carleson measure characterization of identity operators on Hardy spaces of the unit ball of $C^{n}$ using the well-known results of Hormander, and Stegenga [10] has considered similar questions in the unit disc. In this work, which is inspired by the results of Chang and Hastings, we present three theorems which characterize the compact identity operators from Hardy spaces of polydiscs into $L^{p}$ spaces, and the corresponding bounded and compact identity operators on weighted Bergman spaces. As an immediate application of these characterizations we prove Proposition 1.3 and a weighted Fejer-Riesz inequality. Further application of these results to a large class of operators on these spaces are discussed elsewhere [5]. For reference we shall state the result of Chang here. 
Theorem 2.1 (Chang). Let $\mu$ be a positive finite measure on $\overline{U^{n}}$. Then $\mu$ is bounded in $L^{p}\left(T^{n}\right), 1<p<\infty$, i.e.

if and only if

$$
\int_{U^{n}}|P[f]|^{p} d \mu \leq C \int_{T^{n}}|f|^{p} d m_{n}
$$

$$
\mu(S(V)) \leq C m_{n}(V) \text { for all connected open sets } V \subset T^{n} ;
$$

i.e., (1) holds if and only if $\mu$ is a Carleson measure for $H^{p}$.

$P[f]$ in (1) denotes the Poisson integral of the function $f$. In part, the significance of this theorem is explained by this corollary which follows by using a standard perturbation of the proof of Chang.

Corollary 2.2. $\mu$ is bounded in $H^{p}\left(U^{n}\right), 1<p<\infty$, if and only if $\mu$ is a Carleson measure.

The proof of the converse direction of this theorem makes use of the results on nontangential convergence for Poisson integral and the Hardy-Littlewood maximal theorem which are valid for $1<p<\infty$, and thus this proof does not extend to $p=1$. An important consequence of this result is that the boundedness of the measure $\mu$ is evidently independent of the choice of $p$. A similar result holds between the compact Carleson measures on $H^{p}\left(U^{n}\right)$ and the compact identity operators from $H^{p}\left(U^{n}\right)$ into $L^{p}(\mu)$. Precisely,

Theorem 2.3. Let $\mu$ be a finite, nonnegative, Borel measure on $\overline{U^{n}}$ and assume that $H^{p}\left(U^{n}\right) \subset L^{p}(\mu)$. Let $A$ be the identity operator sending $H^{p}\left(U^{n}\right)$ to $L^{p}(\mu), 1<p<\infty$. Then $A$ is a compact operator if and only if $\mu$ is $a$ compact Carleson measure for $H^{p}$, i.e. $\mu$ satisfies

$$
\lim _{\delta \rightarrow 0} \sup _{m_{n}(V)<\delta} \frac{\mu(S(V))}{m_{n}(V)}=0 .
$$

The assumption $H^{p}\left(U^{n}\right) \subset L^{p}(\mu)$ is needed in order for the identity operator to send $H^{p}$ into $L^{p}$.

Proof. First, suppose that $A$ is compact, and to show a contradiction suppose that (1) fails. Then there is an $\varepsilon>0$ and a sequence of open sets $V_{j} \subset T^{n}$ with $\lim _{n} m_{n}\left(V_{j}\right)=0$ so that

$$
\mu\left(S\left(V_{j}\right)\right)>\varepsilon m_{n}\left(V_{j}\right)
$$

It is easy to show that there exists a sequence of rectangles $R_{j} \subset T^{n}$ so that $m_{n}\left(R_{j}\right) \rightarrow 0$ as $j \rightarrow \infty$ for which

$$
\mu\left(S\left(R_{j}\right)\right) \geq \frac{\varepsilon}{2} m_{n}\left(R_{j}\right) .
$$

Say each $R_{j}$ has its center at $\left(e^{i\left(\theta_{1}^{0}+\delta_{1} / 2\right)}, e^{i\left(\theta_{2}^{0}+\delta_{2} / 2\right)}, \ldots, e^{i\left(\theta_{n}^{0}+\delta_{n} / 2\right)}\right)_{j}=\eta_{j}$. Set $\alpha_{j_{k}}=\left(1-\delta_{j_{k}}\right) e^{i\left(\theta_{j}+\delta_{j} / 2\right)_{k}}$, and define $f_{j}$ on $T^{n}$ so that

$$
P\left[f_{j}\right]\left(z_{1}, z_{2}, \ldots, z_{n}\right)=\prod_{i=1}^{n}\left(1-\bar{\alpha}_{i j} z_{i}\right)^{-4 / p} \text {. }
$$


Then

$$
16^{-n} \prod_{i=1}^{n} \delta_{i}^{-4} \mu\left(S\left(R_{j}\right)\right) \leq \int_{S\left(R_{j}\right)}\left|P\left[f_{j}\right]\right|^{p} d \mu \leq \int_{\overline{U^{n}}}\left|P\left[f_{j}\right]\right|^{p} d \mu,
$$

and $\int_{T^{n}}\left|f_{j}\right|^{p} d m_{n} \leq \prod_{i=1}^{n} \delta_{i}^{-3}$. Define $g_{j}=P\left[f_{j}\right] /\left\|f_{j}\right\|_{p}$. Here $\|\cdot\|_{p}$ denotes the $H^{p}$ norm of $f_{j}$. Then $g_{j} \in H^{p}\left(U^{n}\right)$ converges to zero weakly since $\delta_{i} \rightarrow 0$ as $j \rightarrow \infty$, and

$$
\begin{aligned}
\int_{U^{n}}\left|g_{j}\right|^{p} d \mu & \geq 16^{-n} \prod_{i=1}^{n} \delta_{i}^{-4} \frac{\mu\left(S\left(R_{j}\right)\right)}{\prod_{i=1}^{n} \delta_{i}^{-3}}=16^{-n} \prod_{i=1}^{n} \delta_{i}^{-1} \mu\left(S\left(R_{j}\right)\right) \\
& \geq 16^{-n} \frac{\varepsilon}{2} m_{n}\left(R_{j}\right) \prod_{i=1}^{n} \delta_{i}^{-1}=C \varepsilon .
\end{aligned}
$$

Hence, the sequence $g_{j}$ satisfies $\int_{U^{n}}\left|g_{j}\right|^{p} d \mu \geq C \varepsilon$. This contradicts the compactness of $A$.

Conversely, suppose that (1) holds; i.e., suppose that

$$
\lim _{\delta \rightarrow 0} \sup _{m_{n}(V) \leq \delta} \frac{\mu(S(V))}{m_{n}(V)}=0 .
$$

Then given $\varepsilon>0$, there exists a $\delta_{0}>0$ so that for all $\delta<\delta_{0}, V \subset T^{n}$ and $m_{n}(V) \leq \delta$

$$
\mu(S(V)) \leq \varepsilon m_{n}(V) .
$$

We show that $A$ is compact. By Lemma 1.4 it suffices to show that $A$ takes bounded sequence $f_{j}$ converging to zero pointwise in $U^{n}$ to a sequence converging to zero in $L^{p}(\mu)$ norm. Decompose $\mu$ so that $\mu=\mu_{1}+\mu_{2}$, where $\mu_{1}$ is the restriction of $\mu$ to $\left(1-\delta_{0}\right) \overline{U^{n}}$, and $\mu_{2}=\mu-\mu_{1}$. Then

$$
\int_{U^{n}}\left|f_{j}\right|^{p} d \mu=\int_{\overline{U^{n}}}\left|f_{j}\right|^{p} d \mu_{1}+\int_{\overline{U^{n}}}\left|f_{j}\right|^{p} d \mu_{2} .
$$

Since $\mu_{2}<\mu, \mu_{2}$ is a compact Carleson measure whenever $\mu$ is; i.e., $\mu_{2}$ satisfies (5). We claim that $\mu_{2}$ satisfies the Carleson condition (2) in Theorem 2.1 with $C$ replaced by $2 \varepsilon$. To see this claim note that if $V \subset T^{n}$ so that $S(V) \subset N, N=\overline{U^{n}}-\left(1-\delta_{0}\right) \overline{U^{n}}$, the claim is immediate from (5). For an arbitrary $V \subset T^{n}$, decompose $V$ into a union of open sets $V_{j}$ so that $m_{n}\left(V_{j}\right)<$ $\delta_{0}$ and $\sum_{j} m_{n}\left(V_{j}\right) \leq 2 m_{n}(V)$. Then $S\left(V_{j}\right) \subset N$, and $S(V) \cap N=\bigcup_{j=1}^{n} S\left(V_{j}\right)$. Hence

$$
\begin{aligned}
\mu_{2}(S(V)) & =\mu(S(V) \cap N) \\
& \leq \sum_{j} \mu\left(S\left(V_{j}\right)\right) \leq \varepsilon \sum_{j} m_{n}\left(V_{j}\right) \leq 2 \varepsilon m_{n}(V) .
\end{aligned}
$$

Therefore $\mu_{2}(S(V)) \leq 2 \varepsilon m_{n}(V)$ for all $V \subset T^{n}$. Hence $\mu_{2}$ satisfies the Carleson condition (2) in Theorem 2.1 with $C=2 \varepsilon$ and thus

$$
\int_{\overline{U^{n}}}\left|f_{j}\right|^{p} d \mu_{2} \leq 2 \varepsilon \int_{T^{n}}\left|f_{j}\right|^{p} d m_{n} \leq 2 M \varepsilon,
$$


where the second inequality is because $f_{j}$ is a norm bounded family. Hence the integral with respect to $\mu_{2}$ may be made as small as required. But for $\delta_{0}$ fixed, the integral with respect to $\mu_{1}$ can also be made as small as required by choosing $j$ sufficiently large. This is because $f_{j}$ converge to zero uniformly on compact subsets of $U^{n}$.

This result shows that compactness of the operator $A$ is independent of $p$.

We now consider an analogous theorem for the weighted Bergman spaces of polydiscs. For $A_{0}^{2}\left(U^{n}\right)$ Hastings' [4] results imply:

Theorem 2.4 (Hastings). Let $\mu$ be a finite positive measure on $\overline{U^{n}}$. Then there exists a constant $C>0$ so that for $1<p<\infty$

$$
\int_{\bar{U}^{n}}\left|f\left(z_{1}, z_{2}, \ldots, z_{n}\right)\right|^{p} d \mu \leq C \int_{\bar{U}^{n}}\left|f\left(z_{1}, z_{2}, \ldots, z_{n}\right)\right|^{p} d \sigma_{n}
$$

for every $f \in A_{0}^{p}\left(U^{n}\right)$ if and only if there is a constant $C>0$ so that

$$
\mu(S(R)) \leq C \prod_{i=1}^{n} \delta_{i}^{2}
$$

for every set $S(R)$ given by definition (i) at the beginning of this section.

Condition (2) states that $\mu$ is an 0-Carleson measure for $A_{0}^{p}\left(U^{n}\right)$. Stegenga [10] obtains a result of this nature for $A_{\alpha}^{p}(U)$ for arbitrary $\alpha>-1$. MacCluer and Shapiro [8] obtain compactness conditions for the weighted Bergman spaces of the disc. We extend Stegenga and MacCluer-Shapiro's results to polydiscs.

Theorem 2.5. Let $I_{\alpha}: A_{\alpha}^{p}\left(U^{n}\right) \rightarrow L^{p}(\mu), 1<p<\infty, \alpha>-1$ be the identity map. Then

(i) $I_{\alpha}$ is a bounded operator if and only if $\mu$ is an $\alpha$-Carleson measure.

(ii) $I_{\alpha}$ is a compact operator if and only if $\mu$ is a compact $\alpha$-Carleson measure.

Proof. In (i) we show that if $f \in A_{\alpha}^{p}\left(U^{n}\right)$ then

$$
\int_{\overline{U^{n}}}|f|^{p} d \mu \leq C \int_{\overline{U^{n}}}|f|^{p} d \sigma_{n, \alpha}
$$

if and only if

$$
\mu(S(R)) \leq C \prod_{i=1}^{n} \delta_{i}^{2+\alpha} .
$$

To prove (2) suppose that (1) holds for all $f \in A_{\alpha}^{p}\left(U^{n}\right)$. Define

$$
f(z)=\prod_{j=1}^{n}\left(1-\overline{\alpha_{j}} z_{j}\right)^{-(\alpha+2) / p},
$$


where $\alpha_{j}=\left(1-\delta_{j}\right) e^{i\left(\theta_{j}^{0}+\delta_{j} / 2\right)}$. Since on $S(R)|f(z)|^{p}>2^{-(\alpha+2)} \prod_{j=1}^{n} \delta_{j}^{-(\alpha+2)}$,

$$
\int_{U^{n}}|f|^{p} d \mu \geq \int_{S(R)}|f|^{p} d \mu \geq 2^{-(\alpha+2)} \prod_{j=1}^{n} \delta_{j}^{-(\alpha+2)} \mu(S(R)) .
$$

On the other hand, $f(z)$ is clearly in $A_{\alpha}^{p}\left(U^{n}\right)$. Combining (1) and (3) we get $\mu(S(R)) \leq C \prod_{j=1}^{n} \delta_{j}^{\alpha+2}$. Hence $\mu$ is an $\alpha$-Carleson measure. Conversely, to prove (1) suppose that (2) holds for all rectangles in $T^{n}$. Fix $z \in U^{n}$ and let $1-\left|z_{j}\right|^{2}=\delta_{j}$. Consider a polydisc $W_{z}$ centered at $z$ and radius $\delta_{j} / 2$ in the $z_{j}$ coordinate. If $R=I_{1} \times \cdots \times I_{n}$ is the rectangle on $T^{n}$ with $I_{j}$ centered at $z_{j} /\left|z_{j}\right|$ and $\left|I_{j}\right|=2 \delta_{j}$, then $W_{z} \subset S(R)$. Thus for $f \in A_{\alpha}^{p}\left(U^{n}\right)$ the sub-mean-value property for $|f|$ gives

$$
\begin{aligned}
|f(z)| & \leq \frac{C}{\prod_{j=1}^{n}\left(1-\left|z_{j}\right|^{2}\right)^{2}} \int_{W_{z}}|f| d \sigma_{n} \\
& \leq \frac{C}{\prod_{j=1}^{n}\left(1-\left|z_{j}\right|^{2}\right)^{\alpha+2}} \int_{W_{z}}|f| \prod_{j=1}^{n}\left(1-\left|w_{j}\right|^{2}\right)^{\alpha} d \sigma_{n} \\
& \leq \frac{C}{\prod_{j=1}^{n} \delta_{j}^{\alpha+2}} \int_{S(R)}|f| d \sigma_{n, \alpha} .
\end{aligned}
$$

Noting that $\sigma_{n, \alpha}(S(R))=C \prod_{j=1}^{n} \delta_{j}^{2+\alpha}$, we have

$$
|f(z)| \leq \frac{C}{\sigma_{n, \alpha}(S(R))} \int_{S(R)}|f| d \sigma_{n, \alpha} .
$$

This inequality is the heart of the proof. Now define

$$
B_{\alpha}[f](z)=\sup _{R} \frac{1}{\sigma_{n, \alpha}(S(R))} \int_{S(R)}|f| d \sigma_{n, \alpha} .
$$

We have

$$
|f(z)| \leq C B_{\alpha}[f](z) .
$$

In addition, by hypothesis $\mu(S(R)) \leq C \sigma_{n, \alpha}(S(R))$. Using a natural generalization of the argument used for getting the weak type $(1,1)$ inequality (for example, as in Theorem 9.4 [2]), we show that there exists a constant $C$ independent of $s$ so that

$$
\mu\left\{B_{\alpha}[f]>s\right\} \leq C s^{-1}\|f\|_{1, \alpha} .
$$

Given (6), since $B_{\alpha}$ is obviously a sublinear operator of type $(\infty, \infty)$, by the Marcinkiewicz interpolation theorem $B_{\alpha}$ is a bounded operator from $L^{p}\left(\sigma_{n, \alpha}\right)$ into $L^{p}(\mu)$ for $p>1$. Thus

$$
\int_{\overline{U^{n}}}\left|B_{\alpha}[f]\right|^{p} d \mu \leq C \int_{\overline{U^{n}}}|f|^{p} d \sigma_{n, \alpha} .
$$


Using (5) and (7) we conclude

$$
\begin{aligned}
\int_{\overline{U^{n}}}|f|^{p} d \mu & \leq C \int_{\overline{U^{n}}}\left|B_{\alpha}[f]\right|^{p} d \mu \quad(\text { by }(5)) \\
& \leq C \int_{\overline{U^{n}}}|f|^{p} d \sigma_{n, \alpha}=C\|f\|_{p, \alpha}^{p} .
\end{aligned}
$$

This proves (1). To complete the proof we must prove (6); i.e., we need to show that if $\mu(S(R)) \leq C \sigma_{n, \alpha}(S(R))$ then $\mu\left\{B_{\alpha}[f]>s\right\} \leq C s^{-1}\|f\|_{1, \alpha}$. Let $R_{z}=I_{1} \times \cdots \times I_{n}$ denote a rectangle on $T^{n}$ with $I_{j}$ denoting intervals centered at $z_{j} /\left|z_{j}\right|$ and radius $\left(1-\left|z_{j}\right|\right) / 2$. Let $S_{z}$ denote the corona associated with $R_{z}$. Note that $z \in S_{z}$. Define

$$
A_{s}^{\varepsilon}=\left\{z \in U^{n}: \int_{S_{z}}|f| d \sigma_{n, \alpha}>s\left(\varepsilon+\sigma_{n, \alpha}\left(S_{z}\right)\right)\right\},
$$

and note that $\Lambda=\left\{z \in U^{n}: B_{\alpha}[f]>s\right\}=\bigcup_{\varepsilon>0} A_{s}^{\varepsilon}$, i.e. $\mu(\Lambda)==\lim _{\varepsilon \rightarrow 0} \mu\left(A_{s}^{\varepsilon}\right)$. Further note that if $z \in A_{s}^{\varepsilon}$ and $S_{z}$ are disjoint for the different $z \in \Lambda$, then by $(8)$

$$
s \sum_{z \in \Lambda}\left(\varepsilon+\sigma_{n, \alpha}\left(S_{z}\right)\right)<\sum_{z \in \Lambda} \int_{S_{z}}|f| d \sigma_{n, \alpha} \leq\|f\|_{1, \alpha} .
$$

Therefore we have

$$
s \sum_{z \in \Lambda}\left(\varepsilon+\sigma_{n, \alpha}\left(S_{z}\right)\right) \leq\|f\|_{1, \alpha} .
$$

In particular, (9) shows that there are only finitely many $z \in A_{s}^{\varepsilon}$ so that their corresponding $S_{z}$ are disjoint. From these extract the points, $z_{1}, \ldots, z_{l}$, that in addition have the property that if their associated $S_{z}$ radii are multiplied by five in each coordinate the resulting sets cover $A_{s}^{\varepsilon}$. This follows from a standard covering lemma. Write the $S_{z}$ associated with these points as $S_{1}, \ldots, S_{l}$. Since $A_{s}^{\varepsilon} \subset \bigcup_{k=1}^{l} 5 S_{k}, S_{k}$ are pairwise disjoint,

$$
\mu\left(A_{s}^{\varepsilon}\right) \leq 5^{n} \sum_{k=1}^{l} \mu\left(S_{k}\right)
$$

Also, since by hypothesis

$$
\mu\left(S_{z}\right) \leq C \sigma_{n, \alpha}\left(S_{k}\right),
$$

combining (10), (11), and (9) gives

$$
\begin{aligned}
s \mu\left(A_{z}^{\varepsilon}\right) & \leq 5^{n} s \sum_{k=1}^{l} \mu\left(S_{k}\right) \\
& \leq C s \sum_{k=1}^{l}\left(\varepsilon+\sigma_{n, \alpha}\left(S_{k}\right)\right) \leq C\|f\|_{1, \alpha} .
\end{aligned}
$$


Allowing $\varepsilon$ to tend to zero we conclude with

$$
\mu\left\{z \in U^{n}: B_{\alpha}[f]>s\right\} \leq C s^{-1}\|f\|_{1, \alpha} .
$$

This is the desired inequality in (6). Thus the proof is complete.

(ii) Suppose that $I_{\alpha}$ is a compact operator from $A_{\alpha}^{p}\left(U^{n}\right)$ into $L^{p}(\mu)$. Let

$$
f_{\delta}\left(z_{1}, z_{2}, \ldots, z_{n}\right)=\prod_{i=1}^{n} \frac{\delta_{i}^{\beta-(\alpha+2) / p}}{\left(1-\overline{\eta_{i}} z_{i}\right)^{\beta}},
$$

where $0<\delta_{j}<1, \beta>(\alpha+2) / p$, and $\eta_{j}=\left(1-\delta_{j}\right) e^{i\left(\theta_{j}^{0}+\delta_{j} / 2\right)}$. These functions form a bounded subset of $A_{\alpha}^{p}\left(U^{n}\right)$, and tend to zero weakly as $\delta_{i} \rightarrow 0$. Since $\left|1-\bar{\eta}_{i} z_{i}\right|<2 \delta_{i}$, on regions $S(R)$,

$$
\begin{aligned}
\left|f_{\delta}\left(z_{1}, z_{2}, \ldots, z_{n}\right)\right|^{p} & >\prod_{i=1}^{n} \frac{\delta_{i}^{(\beta-(\alpha+2) / p)^{p}}}{\left(2^{\beta} \delta_{i}^{\beta}\right)^{p}} \\
& =\prod_{i=1}^{n} \frac{1}{\delta_{i}^{\alpha+2} 2^{p \beta}} .
\end{aligned}
$$

Hence

$$
\frac{\mu(S(R))}{\prod_{i-1}^{n} 2^{p \beta} \delta_{i}^{\alpha+2}} \leq \int_{\overline{U^{n}}}\left|f_{\delta}\right|^{p} d \mu=: \varepsilon(\delta),
$$

where $\varepsilon(\delta) \rightarrow 0$ as $\delta_{i} \rightarrow 0$ for some $i$. Hence $\mu(S) \leq \varepsilon(\delta) 2^{n \beta p} \prod_{i=1}^{n} \delta_{i}^{\alpha+2}$; i.e.

$$
\lim _{\delta_{i} \rightarrow 0} \sup _{\theta \in T^{n}} \frac{\mu(S(R))}{\prod_{i=1}^{n} \delta_{i}^{\alpha+2}}=0 .
$$

Hence $\mu$ is a compact $\alpha$-Carleson measure for $A_{\alpha}^{p}\left(U^{n}\right)$. Conversely, suppose that $\mu$ is a compact $\alpha$-Carleson measure. We want to show that $I_{\alpha}$ is compact. By Lemma 1.4 it is sufficient to show that if $f_{j} \in A_{\alpha}^{p}\left(U^{n}\right)$ converges to zero weakly, as members of $L^{p}(\mu)$, then the sequence $f_{j}$ converges to zero in norm. The hypothesis on $\mu$ states that given $\varepsilon>0$ we can choose $\delta$ so small that

$$
\sup _{\theta \in T^{n}} \frac{\mu(S(R))}{\prod_{i=1}^{n} \delta_{i}^{\alpha+2}} \leq \varepsilon(\delta)
$$

for all $\delta_{i}<\delta$. Fix $\varepsilon>0$ and choose $\delta$ so that (12) is satisfied. Write $\mu=\mu_{1}+\mu_{2}$, where $\mu_{1}$ is the restriction of $\mu$ to $(1-\delta) \overline{U^{n}}$ and $\mu_{2}$ lives on the complement of this set in $\overline{U^{n}}$. Then, since $\mu_{2} \leq \mu$,

$$
\sup \frac{\mu_{2}(S(R))}{t^{n(\alpha+2)}} \leq \sup \frac{\mu(S(R))}{t^{n(\alpha+2)}},
$$

where the supremums are extended over all $\theta \in T^{n}$ and for all $0<t<\delta$. Since $\mu$ is a compact $\alpha$-Carleson measure, by (12) the right-hand side of the above expression is less than $\varepsilon$, i.e. $\mu_{2}$ is a compact Carleson measure (for $A_{\alpha}^{p}$ ). 
Proceeding exactly as in Theorem 2.3 we note that $\mu_{2}$ satisfies the Carleson condition (for $A_{\alpha}^{p}$ ) in part (i) with the constant $C$ replaced by $C \varepsilon(\delta)$. Hence

$$
\int_{\bar{U}^{n}}\left|f_{j}\right|^{p} d \mu_{2} \leq \sup \frac{\mu_{2}(S(R))}{t^{n(\alpha+2)}}\left\|f_{j}\right\|_{p, \alpha}^{p} \leq C \varepsilon(\delta)\left\|f_{j}\right\|_{p, \alpha}^{p} .
$$

Since $f_{j}$ is a norm bounded family, the integral with respect to $\mu_{2}$ can be made as small as desired. For $\delta$ fixed, the integral with respect to $\mu_{1}$ can also be made arbitrarily small by choosing $j$ sufficiently large.

Theorem 2.5 can be generalized to a relation between the identity operator $I_{\alpha}: A_{\alpha}^{p}\left(U^{n}\right) \rightarrow L^{q}(\mu), 1<p \leq q<\infty$, and the measure $\mu$. That is, it is possible to show that $I_{\alpha}$ is bounded if and only if $\mu(S(R)) \leq C \prod_{i=1}^{n} \delta_{i}^{q(\alpha+2) / p}$, and likewise $I_{\alpha}$ is compact if and only if an analogous statement is true, with $C$ replaced by $\varepsilon$. Since the Carleson condition is independent of $p$, and by part (iv) of Corollary $1.2 A_{-1}^{2}\left(U^{n}\right)=H^{2}\left(U^{n}\right)$, we find that $I_{\alpha}: H^{p} \rightarrow L^{p}(\mu)$ is bounded if and only if $\mu(S(R)) \leq C \prod_{i=1}^{n} \delta_{i}=C m_{n}(R)$, and $I_{\alpha}$ is compact if and only if the above inequality holds with $C$ replaced by $\varepsilon$. This is of course the same as Corollary 2.2. We conclude this section by giving two applications of Theorem 2.5. First, we shall use Theorem 2.5 to prove Proposition 1.5, and then we shall obtain a weighted Fejer-Riesz inequality.

Proof of Proposition 1.3. By Theorem 2.5 it is sufficient to prove the assertion for $p=2$. By Corollary $1.2, A_{\alpha}^{2} \subset A_{\beta}^{2}$ whenever $-1<\alpha<\beta$. Suppose $I_{\alpha}$ is bounded on $A_{\alpha}^{2}$. Then by Theorem 2.5(i), $\mu_{\alpha}$ is an $\alpha$-Carleson measure. Hence

$$
\mu_{\alpha}(S(R)) \leq C \prod_{i=1}^{n} \delta_{i}^{\alpha+2}
$$

$S(R)$ here is the same as in definition (i) at the beginning of this section. Fix $S(R)=S$. On $S, 1-\left|z_{j}\right|^{2} \leq \delta_{j}$, so we have

$$
\begin{aligned}
\mu_{\beta}(S) & =\int_{S} \prod_{i=1}^{n}\left(1-\left|z_{i}\right|^{2}\right)^{\beta} d \nu_{n} \\
& \leq \prod_{i=1}^{n} \delta_{i}^{\beta-\alpha} \int_{S} \prod_{i=1}^{n}\left(1-\left|z_{i}\right|^{2}\right)^{\alpha} d \nu_{n} \\
& =\prod_{i=1}^{n} \delta_{i}^{\beta-\alpha} \mu_{\alpha}(S) .
\end{aligned}
$$

Hence

$$
\mu_{\beta}(S) \leq \prod_{i=1}^{n} \delta_{i}^{\beta-\alpha} \mu_{\alpha}(S)
$$

Combining (2) and (1) we get

$$
\mu_{\beta}(S) \leq \prod_{i=1}^{n} \delta_{i}^{\beta-\alpha} \mu_{\alpha}(S) \leq \prod_{i=1}^{n} \delta_{i}^{\beta-\alpha} C \prod_{i=1}^{n} \delta_{i}^{\alpha+2}=C \prod_{i=1}^{n} \delta_{i}^{\beta+2} .
$$


Using Theorem 2.5 once again we conclude that $I_{\beta}$ is a bounded operator on $A_{\beta}^{2}\left(U^{n}\right)$.

To show compactness we replace the constant $C$ by $\varepsilon(\delta)$ throughout the above argument.

Corollary 2.6. If $f \in A_{\alpha}^{p}\left(U^{n}\right)$ and $\alpha>0$, then

$$
\int_{[0,1]^{n}}|f|^{p} \prod_{i=1}^{n}\left(1-r_{i}^{2}\right)^{\alpha+1} d r_{1} \cdots d r_{n} \leq C \int_{U^{n}}|f|^{p} \prod_{i=1}^{n}\left(1-\left|z_{i}\right|^{2}\right)^{\alpha} d \sigma_{n}(z) .
$$

Proof. Let $\mu=\prod_{i=1}^{n}\left(1-r_{i}^{2}\right)^{\alpha+1} d r_{1} \cdots d r_{n}$. Then

$$
\mu(S(R)) \leq C \prod_{i=1}^{n} \delta_{i}^{2+\alpha} .
$$

Hence $\mu$ is an $\alpha$-Carleson measure on $A_{\alpha}^{p}\left(U^{n}\right)$ and thus, by Theorem 2.5,

$$
\int_{\bar{U}^{n}}|f|^{p} d \mu \leq C \int_{\overline{U^{n}}}|f|^{p} \prod_{i=1}^{n}\left(1-\left|z_{i}\right|^{2}\right)^{\alpha} d \sigma_{n}(z),
$$

i.e.

$$
\int_{[0,1]^{n}}|f|^{p} \prod_{i=1}^{n}\left(1-r_{i}^{2}\right)^{\alpha} d r_{1} \cdots d r_{n} \leq C \int_{\bar{U}_{n}}|f|^{p} \prod_{i=1}^{n}\left(1-\left|z_{i}\right|^{2}\right)^{\alpha} d \sigma_{n}(z) .
$$

\section{REFERENCES}

1. S.-Y. A. Chang, Carleson measure on the bi-disc, Ann. of Math. (2) 109 (1979), 613-620.

2. P. L. Duren, Theory of $H^{p}$ spaces, Academic Press, New York, 1970.

3. J. Garnett, Bounded analytic functions, Academic Press, New York, 1980.

4. W. W. Hastings, A Carleson measure theorem for Bergman spaces, Proc. Amer. Math. Soc. 52 (1975), 237-241.

5. F. Jafari, On bounded and compact composition operators in polydiscs, Canad. J. Math. (to appear).

6. _ Composition operators in polydiscs, Dissertation, University of Wisconsin, Madison, 1989.

7. B. D. MacCluer, Compact composition operators on $H^{p}\left(B_{N}\right)$, Michigan Math. J. 32 (1985), 237-248.

8. B. D. MacCluer and J. H. Shapiro, Angular derivatives and compact composition operators on the Hardy and Bergman spaces, Canad. J. Math. 38 (1986), 878-906.

9. W. Rudin, Function theory in the unit ball of $C^{n}$, Springer-Verlag, New York, 1980.

10. D. S. Stegenga, Multipliers of the Dirichlet spaces, Illinois J. Math. 24 (1980), 113-139.

Mathematics Department, Bowdoin College, Brunswick, Maine 04011 